MATEC Web of Conferences 22,05021 (2015)

DOI: $10.1051 /$ matec conf/ 20152205021

(C) Owned by the authors, published by EDP Sciences, 2015

\title{
Structural Design and Simulation of Passive Low Gravity Compensation for the Deployable Arm in the Device of Drilling and Collecting Lunar Soil
}

\author{
Jianguo Tao \& Guoxing Wang* \\ Research Center of Aerospace Mechanism and Control, Harbin Institute of Technology, Harbin, Heilongjiang, \\ China
}

Jianyong Li

Beihua University, Jilin, Jilin, China

\begin{abstract}
As an important constituent part of the sampling device for lunar soil drilling, the deployable arm can help transfer the samples and avoid stretching. According to the ground experimental research requirements for deployable arm, this paper proposed a low-gravity compensation method of passive cam counter weight by establishing a Lagrangian dynamics model of deployable arm. It is emphasized on designing the profile curve of cam mechanism and the mechanical structure of cam component. By using ADAMS software, this paper conducted kinematics analogue simulation and obtained the kinematic parameters of deployable arm under the environmental conditions of the lunar surface and of the ground surface. The comparison of the analogue results has verified the necessity and reasonability of this compensation method. At last, this paper reached the conclusions and proposed measures to improve the compensation device. This paper can offer certain technical reference for the ground experiment of the sampling mechanism used for lunar drilling and the engineering implementation.
\end{abstract}

Keywords: low-gravity compensation, deployable arm, cam mechanism, kinematics simulation

\section{INTRODUCTION}

The moon is the first choice of deep space exploration in China. According to the Three-Step Lunar Exploration Plan of Circling-Landing-Returning, Chang'e 5 detector will start the exploration of lunar soil in the landing area for the first time in about 2017. It will return to the earth with lunar soil samples ${ }^{[1]}$. The lander bring detecting instrument to research on the lunar terrain features and lunar substance in the detection region. It will also detect the spatial environment of lunar base and space weather, obtaining the background information of the detection region and selecting proper locations to drill for sampling. The implementation of phase III lunar exploration engineering will deepen our understanding of the formation and evolution of lunar soil, lunar crust and the moon, and can provide data support for the follow-up project of lunar exploration ${ }^{[2]}$.

After designing the device of drilling and collecting lunar soil, the equivalent simulation of the low gravity environment on lunar surface needs to be conducted in the ground test phase and the gravity compensation shall be added in the stretching motion of the deployable arm ${ }^{[3]}$. The gravity compensation devices which have been studied at home and overseas are mainly used for space manipulator, cosmonaut ground simulation experiment, and extraterrestrial celestial body detector.The method of gravity compensation is

*Corresponding author: wangguoxing1990@sina.com mainly related to the air-floating platform ${ }^{[4]}$, the water-floating method ${ }^{[5]}$ and the active suspension control ${ }^{[6-7]}$. However, it can be concluded from comparison that all these common methods are developed for certain needs. They all have some defects and limited conditions. According to the stretching motions made by deployable arm on the lunar surface, this paper designed a passive low gravity compensation device based on the cam mechanism counter weight method. By changing the length of counter weight force arm through the cam profile curve, the compensation of balanced force provided by this device can remain constant. The counter weight torque can compensate the gravitational torque at real time in full course.

\section{LOW-GRAVITY COMPENSATION METHOD OF DEPLOYABLE ARM}

\subsection{Kinetic model of deployable arm}

As an important constituent part of the sampling apparatus for lunar soil, the deployable arm can provide support and transport for the lunar soil samples in soft bags from drilling lunar soil to the device of shaping and transmitting samples. When the samples are transferred to the sealing container of rising explorer, the deployable arm that connected to the shaping mechanism will be unlocked and released, so as to ensure the rising explorer with lunar soil 
samples can return to the earth smoothly. It will be fastened again after rotating to a certain angle under the power of the torque spring, so as to provide enough space for the rising of the rising explorer. The schematic diagram of the deployable arm working status is shown in Figure $1^{[8]}$.

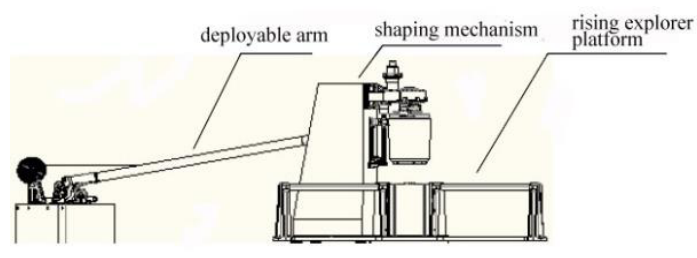

(a)

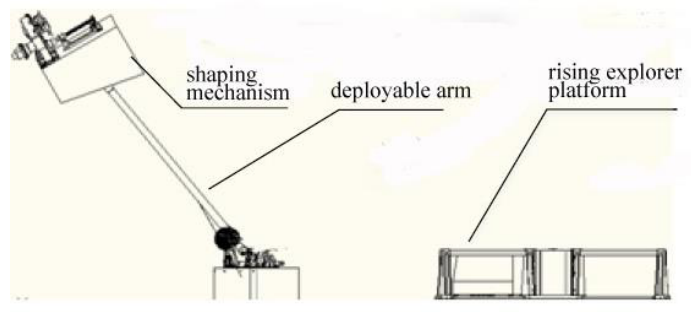

(b)

Figure 1. Sampling state of deployable arm

The stretching motion of the deployable arm is the accelerating circling motion of the rotary center. The Figure 2 is shown for the stress analysis in a simplified model. In this figure, the length of deployable arm is L. Ignore the distance from the deployable arm end to the loading centroid, then the distance from the loading centroid to the rotary center equals L.The distance from the deployable arm centroid to the rotary center equals $1 / 2 \mathrm{~L} . \mathrm{m}_{1}$ and $\mathrm{m}_{2}$ respectively refer to the quality of the deployable arm and the quality of the load. $v_{1}$ and $v_{2}$ respectively refer to the linear speeds. $\mathrm{K}_{0}$ refers to the elastic coefficient of the torque spring in the rotary center. $\theta_{0}$ refers to the initial angle of spring pre-tightening. $\theta$ refers to the horizontal contained angle of the deployable arm.

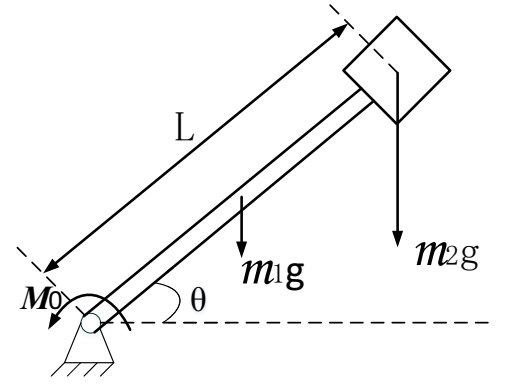

Figure 2. Mechanics analysis chart of deployable arm
Apply the Lagrangian method to complete dynamic modeling in the lunar surface environment. The kinetic energy of the system is shown as follows:

$$
\begin{aligned}
& \mathrm{T}=\frac{1}{2} m_{1} v_{1}^{2}+\frac{1}{2} m_{2} v_{2}^{2}=\int_{0}^{l} \frac{1}{2} \frac{m_{1}}{L}(\dot{\theta} x)^{2} d x+\frac{1}{2} \mathrm{~m}_{2}(\dot{\theta} L)^{2} \\
& \mathrm{~T}=\frac{1}{2} L^{2} \dot{\theta^{2}}\left(\frac{m_{1}}{3}+m_{2}\right)
\end{aligned}
$$

The potential energy of the system is shown as follows:

$V=\int_{0}^{L} \frac{m_{1}}{L} \frac{g}{6} x \sin \theta d x+m_{2} \frac{g}{6} \sin \theta \cdot L+\frac{1}{2} K_{0}\left(\theta_{0}^{2}-\theta^{2}\right)$

$V=\frac{1}{6} g \sin \theta \cdot L\left(\frac{m_{1}}{2}+\mathrm{m}_{2}\right)+\frac{1}{2} K_{0}\left(\theta_{0}^{2}-\theta^{2}\right)$

Then, the Lagrangian function can be expressed by the equation given as follows:

$\mathrm{L}=\mathrm{T}-V=\frac{1}{2} L^{2} \dot{\theta}^{2}\left(\frac{m_{1}}{3}+m_{2}\right)$

$-\frac{1}{6} g \sin \theta \cdot L\left(\frac{m_{1}}{2}+\mathrm{m}_{2}\right)-\frac{1}{2} K_{0}\left(\theta_{0}^{2}-\theta^{2}\right)$

Select the $\theta$ which contacts the deployable arm and the horizontal as the generalized coordinate, then:

$\frac{\partial L}{\partial \theta}=-\frac{1}{6} g L \cos \theta\left(\frac{m_{1}}{2}+\mathrm{m}_{2}\right)+K_{0} \theta$

$\frac{\mathrm{d}}{\mathrm{dt}}\left(\frac{\partial L}{\partial \dot{\theta}}\right)=\ddot{\theta} L^{2}\left(\frac{m_{1}}{3}+m_{2}\right)$

As the corresponding non-potential force is zero, the kinetic equation of the deployable arm is shown as follows:

$$
\begin{aligned}
& \frac{\mathrm{d}}{\mathrm{dt}}\left(\frac{\partial L}{\partial \dot{\theta}}\right)-\frac{\partial L}{\partial \theta}=\ddot{\theta} L^{2}\left(\frac{m_{1}}{3}+m_{2}\right) \\
& +\frac{1}{6} g L \cos \theta\left(\frac{m_{1}}{2}+\mathrm{m}_{2}\right)-K_{0} \theta=0
\end{aligned}
$$

\subsection{Composition of the cam counter weight compen- sation device}

The cam counter weight compensation device of the deployable arm is composed of cam mechanism, cam holder, balancing weight, and steel wire rope as shown in Figure 3. As a core part of the device, the cam mechanism is the key in design. The cam mechanism is made up of two profile sections in parallel connection. The inner and outer sides are cam concave slabs while the middle part is composed of several fixed pulleys with bearings. The function of the cam mechanism leads the steel wire rope to smoothly pass through the device and ensures that the steel wire rope 
and the pulleys inside the mechanism can stay in a tangent state during the stretching motion of the deployable arm. Lifting in real time can be guaranteed in this way. The profile curve of the cam mechanism is obtained by the change of the compensational arm of force. The quality of the balancing weight is fixed. It can provide compensational power. The damping spring above the balancing weight can help buffer vibration. One end of the steel wire rope is connected with damping while the suspension spot of the other end lies on the centroid of the shaping mechanism. The cam holder can provide support. While in designing, the spatial installation positions of the cam holder and the detector platform shall be reasonable to avoid interference.

\subsection{Passive low-gravity compensation principle of cam}

The passive compensation is different from the active compensation. The difference between the active-control gravity compensation system and the passive compensation system is that the tensile force used to offset gravity comes from electric motor in active-control gravity compensation system. However, the compensation is obtained by the passive following of the balancing weight during the motion of the compensation object in passive suspension gravity compensation system ${ }^{[9]}$. The low-gravity compensation based on cam counter weight is a method of torque compensation. Figure 3 is shown for the schematic diagram of the collision prevention and stretching motions of the deployable arm. Take the rotating shaft of hinge in the end of the deployable arm as the center. $\mathrm{L}_{1}$ refers to the gravity arm caused by $\mathrm{G}$ and $\mathrm{L}_{2}$ refers to the compensation force arm caused by the tensile force of the steel wire rope $F_{2}$. Regard the deployable arm and the load as a firmly connected rigid body. Use $G$ to present the gravity born by the deployable arm and the load. Use the red imaginary line to present the mass center curve synthesized by the deployable arm and the load during stretching motion, the related equation is given as follows:

$G \times L_{1}=g L \cos \theta\left(\frac{m_{1}}{2}+\mathrm{m}_{2}\right)$

Set $\mathrm{M}_{2}$ as the compensation torque.then $\mathrm{M}_{2}$ is the non-potential force in the ground surface environment after installing compensation device. The Lagrangian dynamical equation of the system is as follows:

$$
\begin{aligned}
& \frac{\mathrm{d}}{\mathrm{dt}}\left(\frac{\partial L}{\partial \dot{\theta}}\right)-\frac{\partial L}{\partial \theta}=\ddot{\theta} L^{2}\left(\frac{m_{1}}{3}+m_{2}\right) \\
& +g L \cos \theta\left(\frac{m_{1}}{2}+\mathrm{m}_{2}\right)-k_{0} \theta=M_{2}
\end{aligned}
$$

From Equation (6) and Equation (8), the compensation torque can be expressed as follows:

$$
M_{2}=F_{2} \times L_{2}=\frac{5}{6} g L \cos \theta\left(\frac{m_{1}}{2}+\mathrm{m}_{2}\right)=\frac{5}{6} G \times L_{1}
$$

\section{MECHANISM DESIGN}

\subsection{Design of came mechanism profile curve}

Figure 4 is shown for the motion trail and mechanics analysis of the center of load contacted with deployable arm. Take the rotary center of the deployable arm hinge as the origin of coordinates $\mathrm{O}$. The polar coordinates solution method used for the cam profile curve is to determine the position of the cam profile curve by the distance from one point on the profile to the rotary center $L_{3}$ and the horizontal angular separation of that point $\xi$.

In the first position, $G$ refers to the synthesized gravity of the load and the arm while $\mathrm{L}_{1}$ refers to the gravity arm. At the moment, the tensile force of the steel wire rope is $F_{2}$ and the force arm of the tensile force is $\mathrm{L}_{2}$. The second position shares the same principle. As the direction of tensile force shall be tangent to the compensation force arm in real time and $\mathrm{P}$, the intersection point of the first position and the secondary position, shall satisfy the tensile force directions in adjacent positions during stretching, $\mathrm{P}$ shall be regarded as the cam profile point. $\mathrm{L}_{3}$ refers to the length of OP and $\xi$ refers to the horizontal angular separation of OP. Moreover, $\theta$ refers to the horizontal angular separation of the deployable arm; $\beta$ refers to the included angle of L2 and OP; $\alpha$ refers to the included angle of L3 and OP. From Equation (9), we can know that the compensation torque during motion equals $5 / 6$ gravity torque. Taking $5 / 6$ weight of load as the farmar gravity and combining the geometrical relations in the figure, we can obtain the relations as follows:

$L_{1}=L_{2}=\cos \theta \times L$

$\xi=2 \theta^{\prime}-\alpha=2 \theta-\beta$

$\cos \alpha=L_{2}^{\prime} / L_{3}$

$\cos \beta=L_{2} / L_{3}$

Set the angle in progressive increase to a certain degree, namely $\theta^{\prime}=\theta+\Delta \theta$, the functions of the cam profile curve are shown as follows:

$$
\cos (\alpha-2 \Delta \theta) / \cos \alpha=\cos \theta / \cos (\theta+\Delta \theta)(14)
$$$$
L_{3}=L \times \cos (\theta+\Delta \theta) / \cos \alpha
$$

$\xi=2(\theta+\Delta \theta)-\alpha$

The function expression of the cam profile curve 


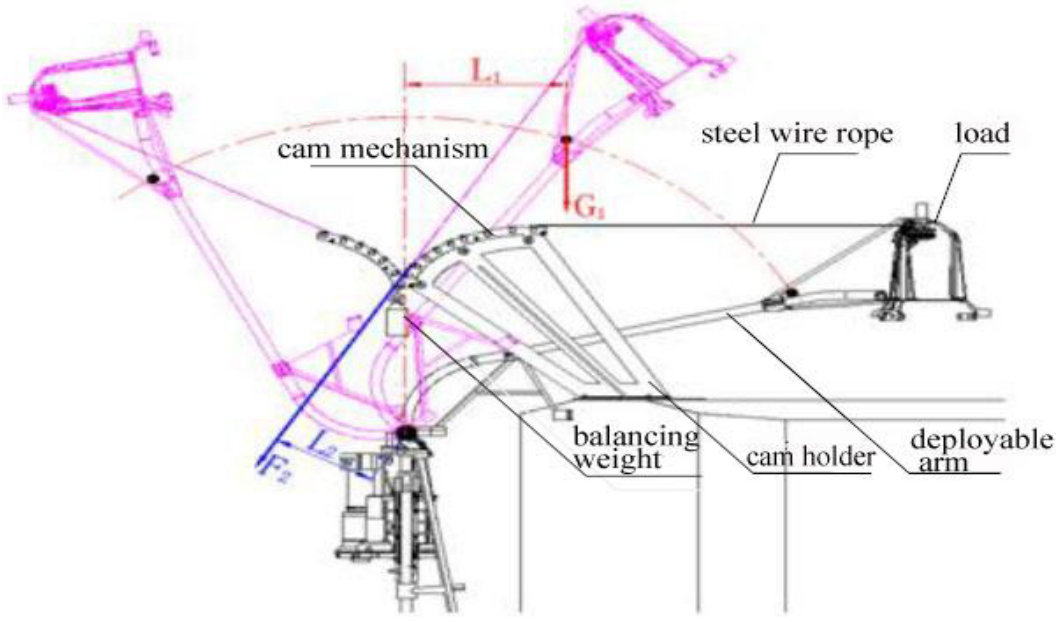

Figure 3. General conceptual drawing of cam passive counter weight

can be obtained by combining the three equations given above.

For example, $\Delta \theta=5^{\circ}$, the precise profile curve shown in Figure 5 can be obtained by software.

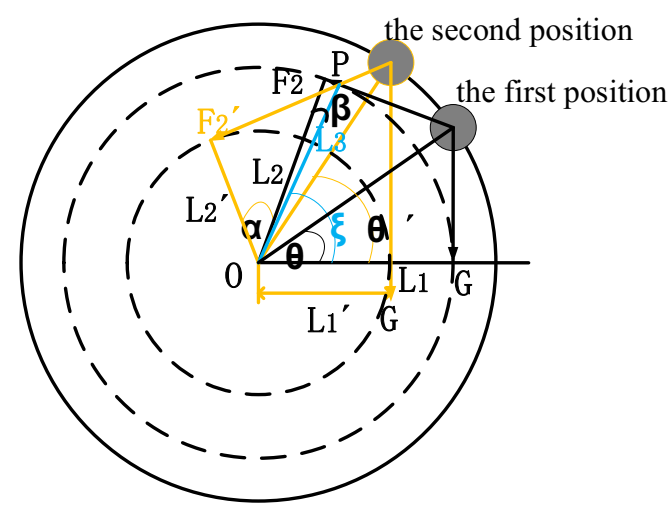

Figure 4. Geometrical relations and mechanics analysis of the center of load contacted with deployable arm

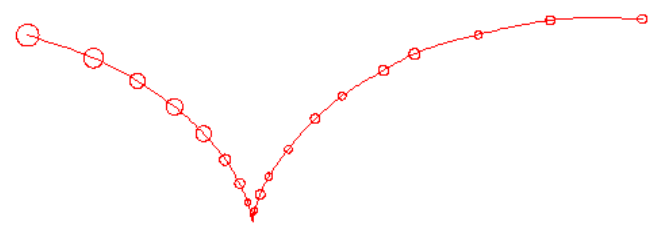

Figure 5. Cam profile curve

\subsection{Design of cam structure}

Use the cam profile curve obtained above to design the groove plates on the inner and outer sides of the cam mechanism. Connect multiple rotary axles along the curve in the grooves and equip the axles with pulleys. The leading sheaves on the lower end can lead the steel wire rope and prevent the rope from slipping out the pulleys. Furthermore, add a bearing mechanism in the pulleys to reduce the friction between the steel wire rope and the cam mechanism. The Figure $6 \mathrm{a}$ is shown for the structural schematic diagram. The counter weight components are formed by spring-dampers and weights. Add spring-dampers to make the process of collision prevention and stretching more stable. Define the total mass of counter-weight components as $\mathrm{M}_{\mathrm{P}}$ and define the mass of compensation object as $\mathrm{M}_{0}$. Start the design by setting $M_{P}=5 / 6 M_{0}$. The Figure $6 b$ is shown as the 3-D model of passive low-gravity cam compensation.

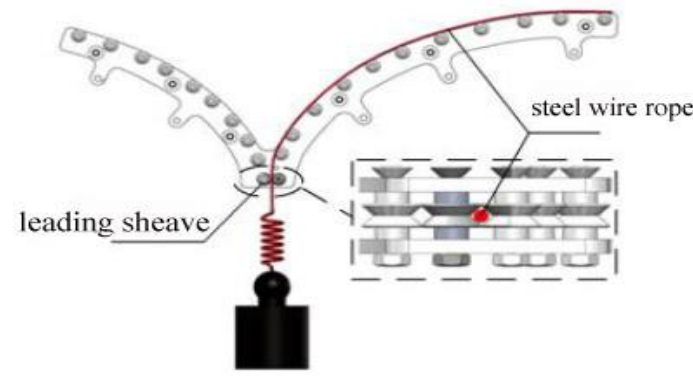

a. Schematic diagram of cam structure

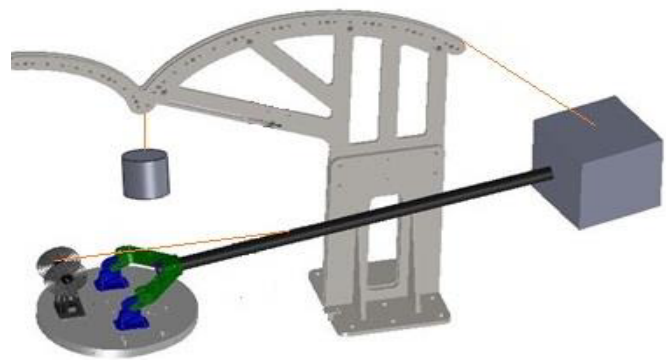

b. Gravity compensation model of cam mechanism

Figure 6. Design of cam structure 


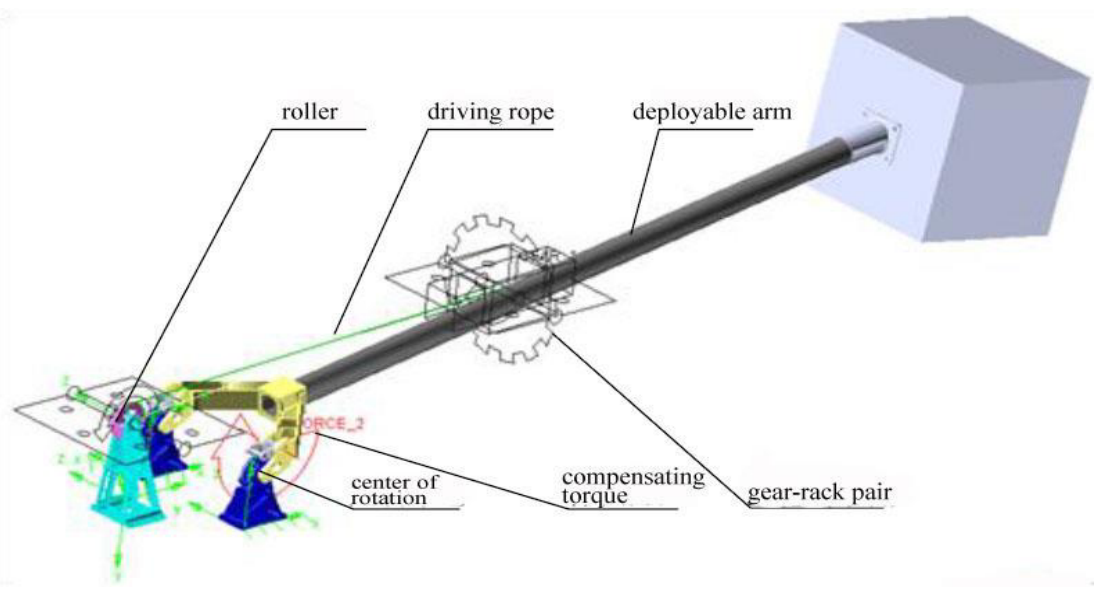

Figure 7. Simulation equivalent model

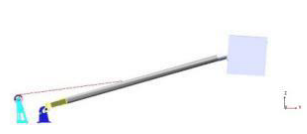

(a) $\mathrm{t}=0.5 \mathrm{~s}$

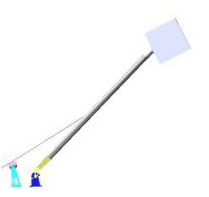

(b) $\mathrm{t}=1.0$

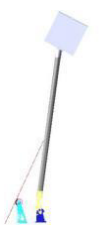

(c) $\mathrm{t}=1.5 \mathrm{~s}$

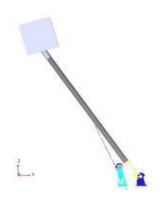

(d) $\mathrm{t}=2.0 \mathrm{~s}$

Figure 8. Motional process of the deployable arm

\section{SIMULATION AND RESULT}

\subsection{Establishment of simulation model}

Establish the three-dimensional model by using solidworks12.0 software. Import the designed model system into ADAMS via the interface of $x-t$ file and ADAMS 2013. Conduct simplifying treatment in the software for the convenience of constructing the physical analysis model of deployable arm which is compensated object. As the explorer and the shaping mechanism will not occur in simulation, no explorer is drawn in the figure. The shaping mechanism is equivalent to the form of heavy piece.

The key of simulation is to set up the kinematic restraint and interactive contact in the geometric model established in the ADAMS environment. It can be seen from the list of system rigid body and revolute pair information that there are 16 rigid bodies, 19 attachment restraints and 19 mechanical contacts. The gravitational field environment shall be individually set up. Moreover, define the material parameters of each part and component one by one so as to make them consistent with the actual situation.

The Figure 7 is a simulation model of the simplified low-gravity compensation. The difficult part of the model is the equivalent simplifying of the rope mechanical transfer process. At the first, the mechanical transfer between the driving rope of deployable arm and the roller connected with volute spiral spring is implemented through the equivalence of gear -rack model. In the actual model, the driving moment is provided by volute spiral spring. The coaxial spring drives the roller to rotate. And then, the roller drives the driving rope to pull up the deployable arm and the rope is tangent with the roller. Linear velocities at the points of tangency are the same. The rope is connected with the deployable arm and starts relative rotation. Therefore, a revolute pair shall be set at the roller while a prismatic pair shall be set at the rope. The application of gear-rack pair in connecting the revolute pair of the roller and the prismatic pair of the rope can remain the synchronous movement of the roller and the rope and keep a same velocities at the points of the tangency. The connection of the rope and the deployable arm which based on the revolute pair can ensure the relative rotation of the roller and the arm. At the second, the mechanical transfer of the driving rope in the compensation device and the loaded barycenter can be equivalent with force moment which is implemented on the rotation center of the deployable arm hinge. The force moment equals $5 / 6$ of the gravity moment. The value of the force moment is negative in order to be in contrast with the gravity moment. Remove the small parts which are irrelevant with the motions before motional simulation. After respectively setted the gravity accelerations of lunar surface field environment and ground environment, the simulation calculation can be started. 
MATEC Web of Conferences

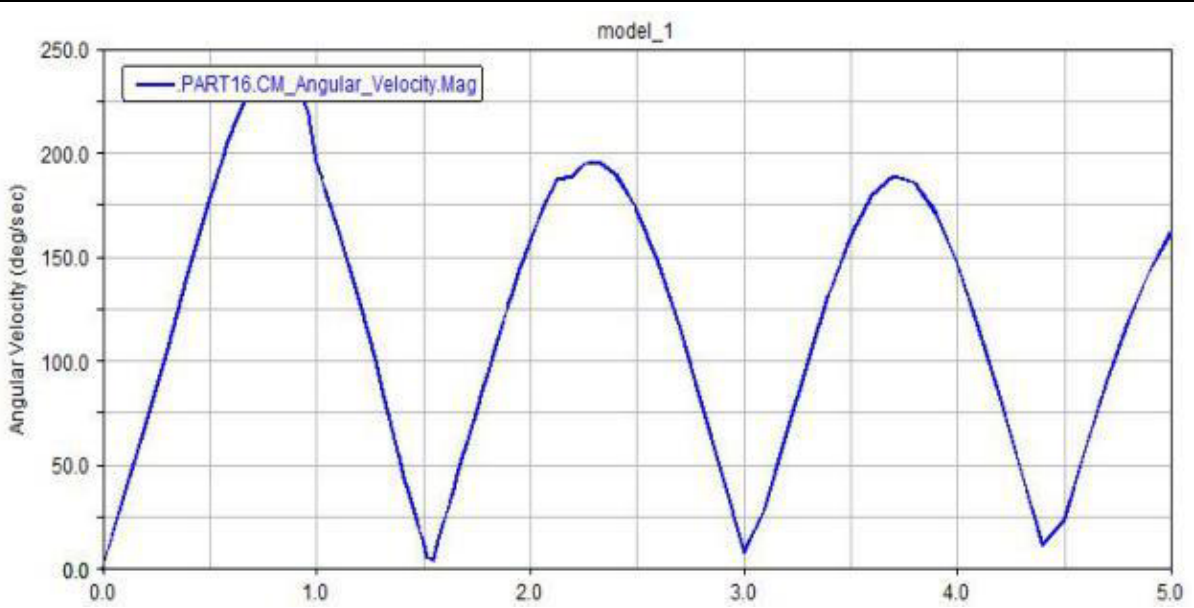

(a) The varying curve of Time-angular velocity on the ground without compensation

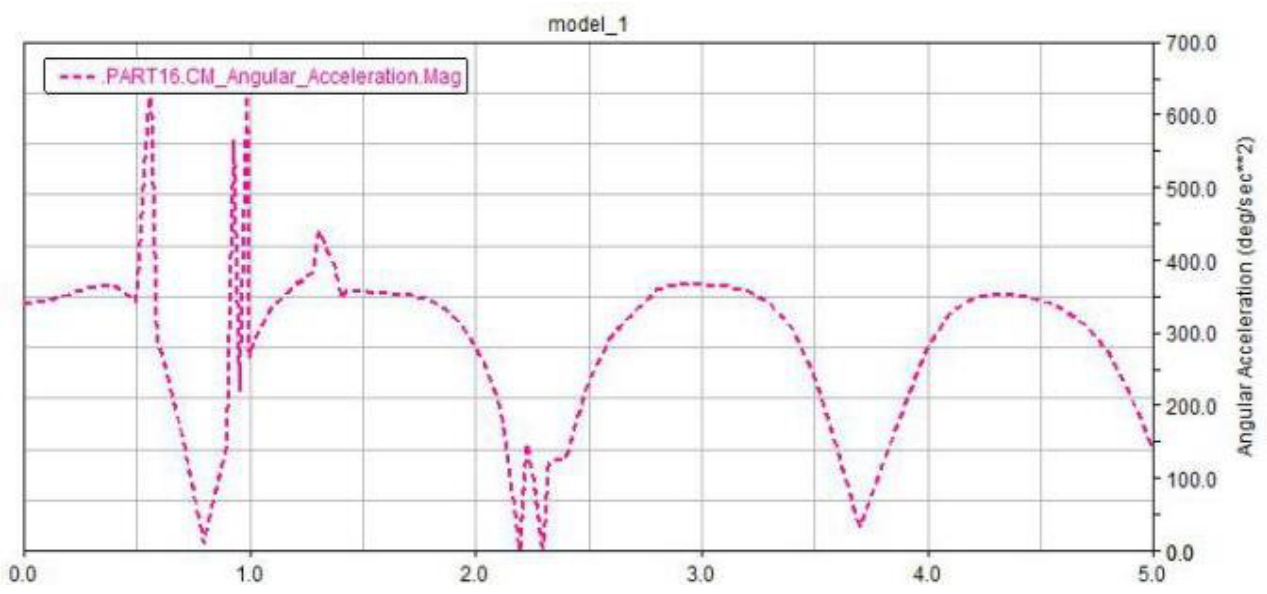

(b) The varying curve of Time-angular acceleration on the ground without compensation

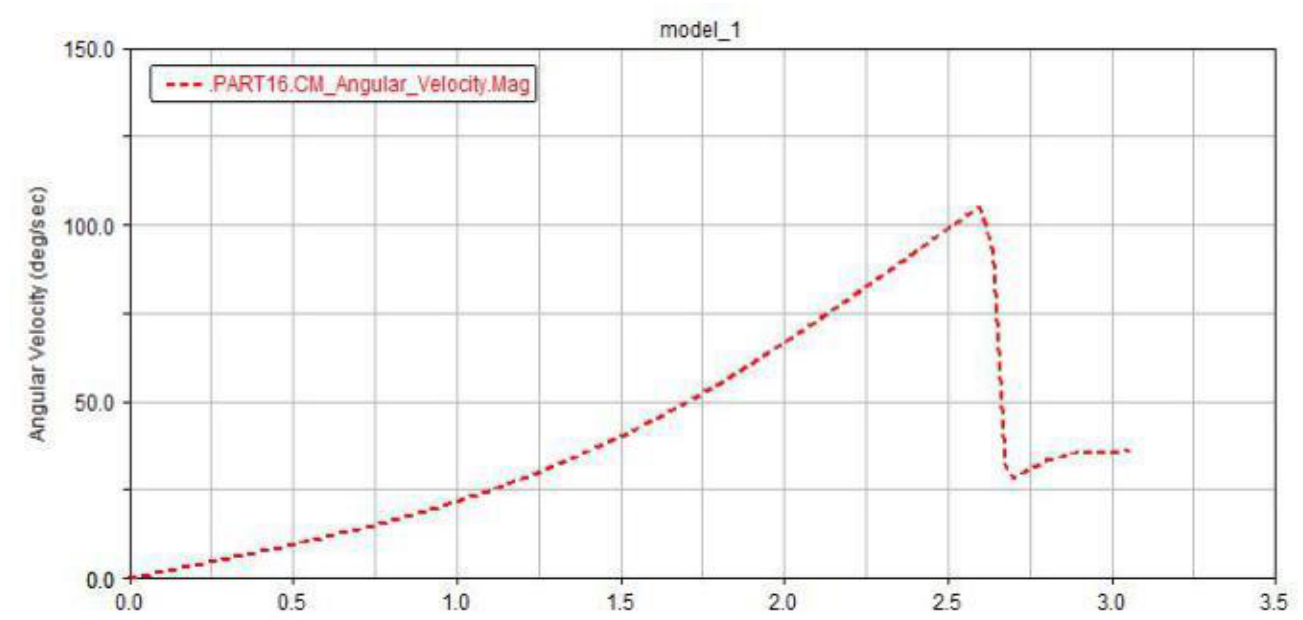

(c) The varying curve of Time-angular velocity on the ground with compensation 
ICETA 2015

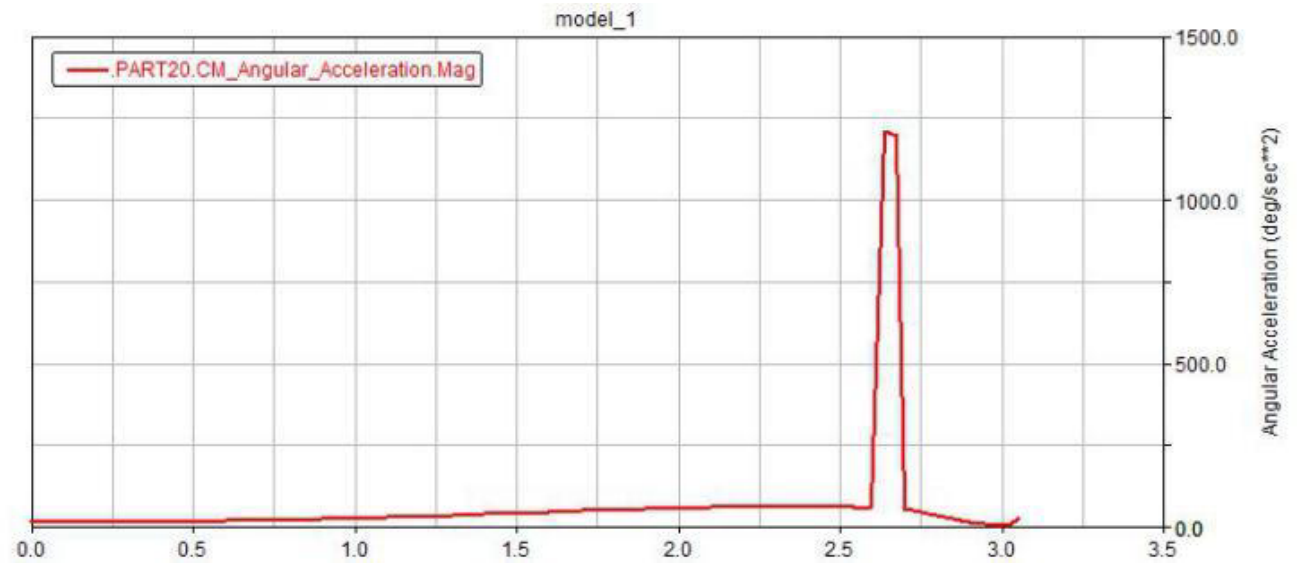

(d) The varying curve of Time-angular acceleration on the ground with compensation

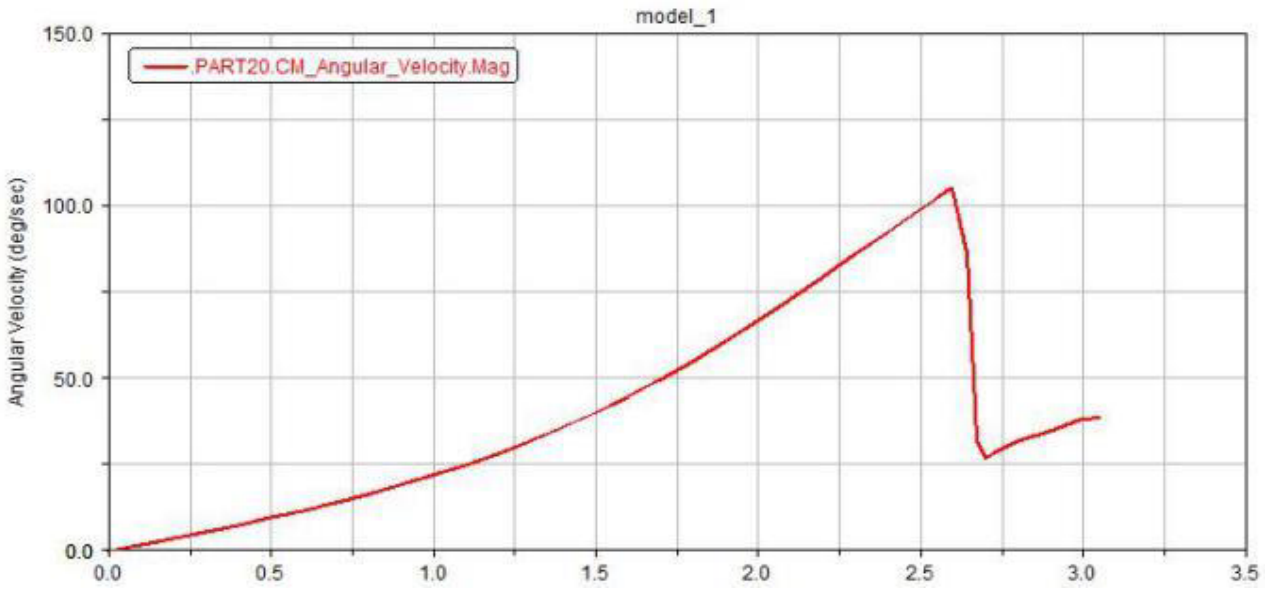

(e) The varying curve of Time-angular velocityon the lunar surface

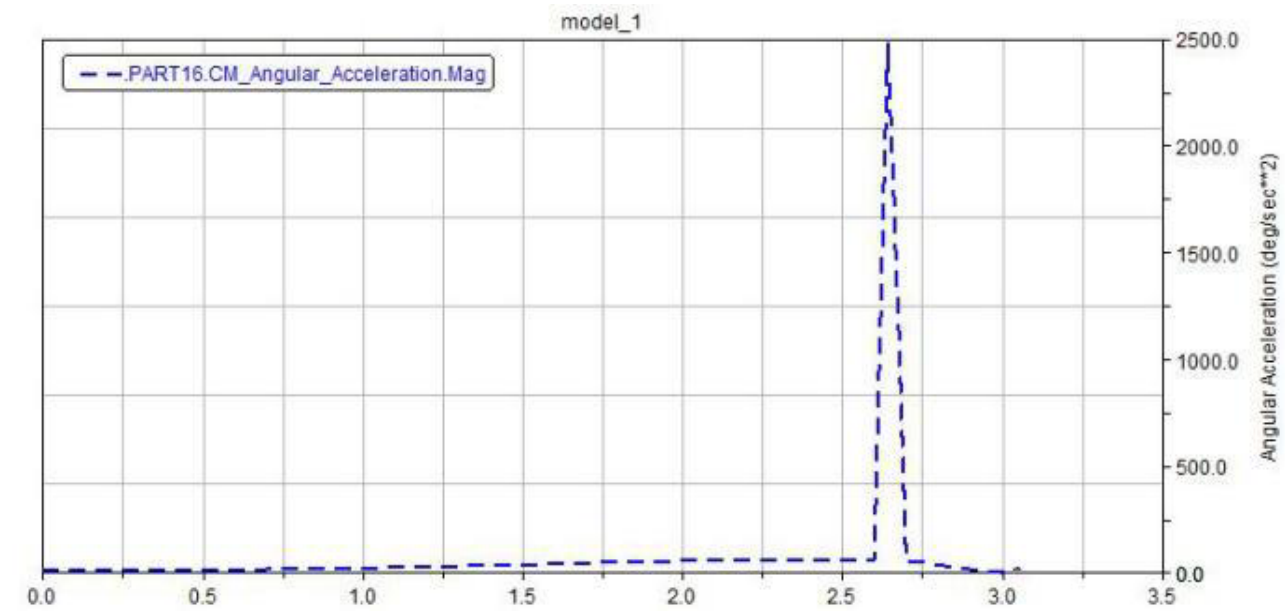

(f) The varying curve of Time-angular acceleration on the lunar surface

Figure 9. Kinematics simulation result of the deployable arm 


\subsection{Result of Simulation}

Take the motion simulation in lunar surface environment as the example. The simulated stretching motional process of the deployable arm is shown in Figure 8 .

Use post-processing software to make data analyses of the motional processes respectively in the ground environment without compensation, in the lunar surface environment and in the ground environment with compensation. The obtained angular velocity and angular acceleration of the deployable arm motion are shown in figure 9 .

Through the comparison of the angular velocity curves and the angular acceleration curves of the deployable arm in the lunar surface environment and in the ground environment without compensation, it can be seen that the deployable arm will rotate to the opposite direction. The curves of angular velocity and angular acceleration both suggest that the deployable arm has reciprocating motion which does not accord with the requirements for working normally on the lunar surface.The results can verify the necessity of low gravity compensation in ground experiment.

Through the comparison of the kinematic parameter curves of the deployable arm in the lunar surface environment and in the ground environment with compensation, it can be seen that the changing trends and values of the angular velocity and the angular acceleration of the deployable arm are similar, the results suggest that the applied compensating measures are reasonable and the compensation precision is ideal.

From the analysis of the two kinds of proper kinematic data, it can be concluded that the angular velocity value rises rapidly while the angular acceleration value stays in a steady state in the initial stage. When the expansion duration of the deployable arm is $2.6 \mathrm{~s}$, the locking slot will take effect. Then the angular velocity will have rapid attenuation and the angular acceleration will have a peak value. At the moment, the maximum angular velocity shall be $105.22 \%$ which is close to the theoretical value, $108.6 \%$ s.

\section{CONCLUSION}

The passive cam counter weight compensation method is used in the low-gravity compensation method for deployable arm. By applying passive mechanical gravity compensation, this method is compact in structure, rapid in reaction, ideal in compensation precision, and high in reliability. Related analyses have obtained the expression of compensating torque via dynamics modeling. Polar coordinates shall be used to obtain the cam profile curve. During simulation, the method of gear-rack can effectively solve the equal effect problem of string simplification. The results of kinematic simulation have verified the consistency of the angular velocity and angular acceleration magnitude of the deployable arm before and after compensation. It can be concluded that the passive cam counter weight method can bring an ideal gravity compensation effect.

At present, the prototype of gravity compensation device has been completed and it is in the trial period. Further improvement needs to be made in two parts: (1) A weight guide rail need to be added and cam brackets need to be optimized in order to avoid the motional interference when the weight is on the state of falling; (2) The optimization of height-direction on the concave boards of the cam is in the plan so as to ensure that the steel wire rope can enter the pulley groove after passing through the pulley smoothly and prevent the rope dropping out from the cam groove on the right side.

\section{ACKNOWLEDGEMENT}

Project fund: funds from The Advanced Research Special Project of Phase III Lunar Exploration (TY3Q20110005) and The Subject Innovation and Intelligence Importing Plan Project for Institution of Higher Education (B07018).

\section{REFERENCES}

[1] Ouyang Z.Y., Li C.L. \& Zhou Y.L., et al 2003. The Progress of Lunar Exploration and the Lunar Exploration of China. Science Foundation in China. (4): 193-197.

[2] Feldman W C, Lawrence D J. \& Elphic R C, et al. 2000. Chemical Information Content of Lunar Thermal and Epithermal Neutrons. Journal of Geophysical Research: Planets (1991-2012), 105(E8): 20347-20363.

[3] Liu Z. 2013. Gravity Compensation for Single Sling of Celestial Body and the Experimental Study. Doctoral Dissertation from Harbin Institute of Technology.

[4] Yoshida K. 1995. Experimental Study on the Dynamics and Control of a Space Robot with Experimental Free-floating Robot Satellite (FFORTS) Simulators. Advanced Robotics, 9(6): 583-602.

[5] Atkins E, Lennon J. \& Peasco R. 2002:215-224. Vision-Based Folmicroing for Cooperative Astronaut Robot Operations. Proceedings of the IEEE Aerospace Conference. Piscataway, NJ, USA: IEEE.

[6] Gao K. 2010. Study of the Ground Gravity Compensation Device for Large-Scale Space Mechanical Arm. Master Dissertation from Harbin Institute of Technology.

[7] Nechyba M C. \& Xu Y S. 1995. Human-robot Cooperation in Space: SM2 for New Space Station Structure. IEEE Robotics\& Automation Magazine, 2(4): 4-11.

[8] Yan W.M. 2014. The Development and Experimental Study on the Branch-Avoiding Mechanism of Lunar Surface Drilling \& Sampling Apparatus. Master Dissertation from Harbin Institute of Technology.

[9] Nechyba M C. \& Xu Y S. 1995. Human-robot Cooperation in Space: SM2 for New Space Station Structure. IEEE Robotics\& Automation Magazine, 2(4): 4-11. 